\title{
Abnormalities on diffusion weighted magnetic resonance imaging performed several weeks after a minor stroke or transient ischaemic attack
}

\author{
U G R Schulz, D Briley, T Meagher, A Molyneux, P M Rothwell
}

J Neurol Neurosurg Psychiatry 2003;74:734-738

See end of article for authors' affiliations

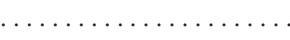

Correspondence to: Dr P Rothwell, Stroke Prevention Research Unit, Department of Clinical Neurology, Radcliffe Infirmary, Woodstock Road, Oxford OX2 6HE, UK;

peter.rothwell@

clneuro.ox.ac.uk

Received 15 August 2002 Accepted in final revised form

15 January 2003

Objectives: Diffusion weighted brain imaging (DWI) is used in acute stroke, and also shows an acute ischaemic lesion in most transient ischamic attack (TIA) patients scanned acutely. However, it may also be useful in identifying subacute ischaemic lesions in patients with minor stroke or TIA who present several weeks after symptom onset. This study investigated the sensitivity and the observer reproducibility of DWI in cerebral TIA and minor ischaemic stroke patients scanned more than two weeks after the last symptomatic event.

Methods: Consecutive patients underwent magnetic resonance imaging (T2, DWI, ADC). The presence of clinically appropriate lesions was assessed by two independent observers, and related to the type of presenting event, the NIH score, persistence of symptoms and signs, and the time since the presenting event.

Results: 101 patients (53 men) were scanned at a median time of 21 days (IQR=17-28) after symptom onset. Reproducibility of the assessment of DWI abnormalities was high: interobserver agreement $=97 \%(\kappa=0.94, p<0.0001)$; intraobserver agreement $=94 \%(\kappa=0.88, p<0.0001)$. DWI showed a clinically appropriate ischaemic lesion in 29 of 51 (57\%) minor stroke patients, and in 7 of 50 (14\%) TIA patients. The independent predictors of a positive DWI scan were presentation with minor stroke versus TIA ( $p=0.009)$ and increasing $\mathrm{NIH}$ score $(p=0.009)$, but there was no difference between patients presenting 2-4 weeks compared with $>4$ weeks after symptom onset. In minor stroke patients, the presence of a clinically appropriate lesion was associated with persistent symptoms $163 \%$ versus $36 \% ; p=0.12)$ and signs $(64 \%$ versus $33 \%, p=0.06)$ at the time of scanning.

Conclusions: DWI shows a clinically appropriate ischaemic lesion in more than half of minor stroke patients presenting more than two weeks after the symptomatic event, but only in a small proportion of patients with TIA. The persistence of lesions on DWI is closely related to markers of severity of the ischaemic event. These results justify larger studies of the clinical usefulness of DWI in subacute minor stroke.

M anagement guidelines recommend that patients with transient ischaemic attack (TIA) and minor stroke are seen as soon as possible after the event, ${ }^{1}$ but many patients do not seek medical attention until several days or even weeks later. ${ }^{2-4}$ In these patients, it may be more difficult to obtain a clear history, and clinical signs may have resolved. Visualisation of a recent ischaemic lesion on brain imaging could therefore help to clarify the diagnosis of a cerebral ischaemic event, and knowledge of the number of lesions, and their territory(s), could also influence management.

Diffusion weighted magnetic resonance imaging (DWI) is a relatively recent imaging technique, which shows ischaemic tissue damage within minutes after onset of the injury. ${ }^{5}$ One of its main uses, therefore, is in the investigation of patients presenting acutely with stroke. ${ }^{6}$ The vast majority of patients with acute ischaemic stroke who are scanned within a few days of the event have an appropriate lesion on DWI, and $50 \%-70 \%$ of patients with TIA have a DWI lesion when scanned within a few hours of onset, ${ }^{7}$ or within a few days of onset. ${ }^{8}$

Serial DWI studies have shown that ischaemic lesions remain visible for at least two weeks after acute stroke, because of the combined effects of the apparent diffusion coefficient (ADC) and T2 effects. ${ }^{9}{ }^{10}$ DWI may therefore also be useful in patients presenting subacutely with cerebral ischaemic events. Only one study of DWI in 53 patients with subacute stroke or TIA has been reported. ${ }^{11}$ However, patients were imaged comparatively early (mean (SD) interval from symptom onset $=6$ (4) days) and none were imaged more than two weeks after the event.

Before undertaking large studies of the clinical usefulness and cost effectiveness of DWI in patients presenting late after a TIA or minor stroke, it is first necessary to determine the frequency with which DWI will detect clinically appropriate ischaemic lesions at the subacute stage, and whether radiological assessment is sufficiently reproducible. We therefore performed DWI in a consecutive series of patients with TIA or minor ischaemic stroke scanned at least two weeks after symptom onset.

\section{METHODS}

We prospectively studied consecutive patients attending an outpatient TIA and minor stroke clinic in a district general hospital in Buckinghamshire, UK, from September 2000 to April 2002. All patients had first presented to their family doctor or hospital emergency department, and were referred to our dedicated stroke clinic for further investigations. This resulted in a delay of several days or sometimes weeks between the patients' first presenting to medical attention and having their MRI scan. Patients were eligible for the study if they had a clinical diagnosis of TIA or non-disabling stroke

Abbreviations: TIA, transient ischaemic attack; DWI, diffusion weighted imaging 
Table 1 Baseline characteristics of 101 patients presenting two weeks or more after a TIA or minor stroke. Absolute numbers and percentages are given for the entire cohort and separately for the 65 patients without and the 36 patients with a lesion on DWI. The percentages refer to the totals in each individual column. The $p$ values were obtained by univariate analysis

\begin{tabular}{lllll}
\hline & $\begin{array}{l}\text { Total } \\
\mathrm{n}=101\end{array}$ & $\begin{array}{l}\mathrm{DWI}- \\
\mathrm{n}=65\end{array}$ & $\begin{array}{l}\mathrm{DWI}+ \\
\mathrm{n}=36\end{array}$ & $\mathrm{p}$ Value \\
\hline Diagnosis stroke & $51(50.5 \%)$ & $22(33.8 \%)$ & $29(80.6 \%)$ & $<0.0001$ \\
Median (IQR) NIH-stroke score & $0(0-1)$ & $0(0)$ & $1.5(0-3)$ & $<0.0001$ \\
Median time (IQR) since symptom onset & $21(17-28)$ & $21(17-28)$ & $21(17-28)$ & 0.581 \\
Male sex & $53(52.5 \%)$ & $34(52.3 \%)$ & $19(52.8 \%)$ & 0.964 \\
Mean (SD) age (y) & $69.8(10.5)$ & $67.8(11.2)$ & $73.3(8.3)$ & 0.010 \\
Hypertension* & $42(41.6 \%)$ & $29(44.6 \%)$ & $13(36.1 \%)$ & 0.406 \\
Diabetes* $_{\text {Hypercholesterolaemia* }}^{*}$ & $7(6.9 \%)$ & $3(4.6 \%)$ & $4(11.1 \%)$ & 0.218 \\
Current smoker & $15(14.9 \%)$ & $13(20.0 \%)$ & $2(5.6 \%)$ & 0.051 \\
Prior TIA & $18(17.8 \%)$ & $9(13.8 \%)$ & $9(25.0 \%)$ & 0.161 \\
Prior stroke & $21(20.8 \%)$ & $18(27.7 \%)$ & $3(8.3 \%)$ & 0.022 \\
History of IHD & $7(6.9 \%)$ & $3(4.6 \%)$ & $4(11.1 \%)$ & 0.218 \\
History of PVD & $23(22.8 \%)$ & $14(21.5 \%)$ & $9(25.0 \%)$ & 0.691 \\
\hline
\end{tabular}

IHD, ischaemic heart disease; PVD, peripheral vascular disease. *Patient receiving treatment for this condition.

and the last symptomatic event had occurred at least two weeks before the clinic. This time cut off was chosen because of the lack of data on the sensitivity of DWI in patients presenting after this time point, and because the ADC is generally thought to return to normal values after this time, with the presence of high signal on DWI then mainly attributable to T2 effects. ${ }^{9}$ In the context of this study we used the term "sensitivity" as the proportion of DWI scans that showed a lesion in the vascular territory suggested by the clinical presentation. Stroke and TIA were defined according to WHO criteria (sudden onset of neurological deficit, persisting for $>24$ hours in case of a stroke, or for $<24$ hours in case of a TIA). ${ }^{12}$ As the aim of the study was to determine the sensitivity of DWI in patients with ischaemic stroke, patients who were found to have a haemorrhagic lesion on MRI were excluded. All ischaemic stroke patients included in the study had had a minor, non-disabling stroke-that is, they were sufficiently well to remain at home after their event, and to attend an outpatient clinic. Baseline clinical data and the NIH Stroke Score ${ }^{13}$ were recorded in all cases. Approval from the local ethics committee was obtained.

The clinic was conducted and data were collected by a consultant neurologist and a neurology trainee. A detailed history was obtained from each patient with a standardised questionnaire. This included date of symptom onset, duration and type of symptoms, number of events, and details on vascular risk factors, past medical history, and medication. All patients underwent a standardised clinical neurological examination, and each patient underwent MRI of the brain on the same day using a 1.5 Tesla Siemens Symphony system with quantum gradients. The study protocol included a axial turbo gradient spin echo sequence (TGSE) (repetition time (TR) $4000 \mathrm{ms,}$ echo time (TE) $95 \mathrm{~ms}$, 19 slices, slice thickness $6.0 \mathrm{~mm}$, matrix $256 \times 256$, FOV $230 \times 230)$ and a diffusion weighted sequence (TR $260 \mathrm{~ms}$, TE $184 \mathrm{~ms}, 20$ slices, slice thickness $6.0 \mathrm{~mm}$, matrix $128 \times 128$, FOV $230 \times 230)$. The diffusion weighted sequence was acquired with three different $b$ values $(b=0$, 500, and $1000 \mathrm{~s} / \mathrm{mm}^{2}$ ). A positive DWI scan was defined as high signal on the b1000 image. In cases with a lesion on DWI, we also reviewed the ADC map and noted whether high signal areas on the b1000 image showed low, high or normal signal on the ADC map when comparing the affected area to the corresponding contralateral area. Furthermore, we assessed whether the lesions present on DWI were also present on the T2 image.

The scans were reviewed independently by a neurologist (PMR) and a neuroradiologist (AM), both with a special interest in cerebrovascular diseases. Neither observer was involved in the patients' care, but they were given a summary of the clinical history and the findings on examination. DWI scans were classified as positive if they showed a lesion that was consistent with the clinical details, and DWI scans with no lesion were classified as negative. As our aim was to determine the sensitivity of DWI scanning in detecting a clinically appropriate lesion, scans with lesions that were felt to be unrelated to the clinical presentation (for example, opposite hemisphere) were also classified as negative for the purposes of this study.

Interobserver reliability of detecting lesions on the DWI was determined between the results of the study neurologist and the study neuroradiologist. In cases of disagreement, the scans were reviewed by both observers together and a consensus was reached for the subsequent analyses. To assess intraobserver reliability, the study neurologist (PMR) re-assessed all scans three months later. In cases with positive DWI scans, both observers also independently reviewed the ADC map and the T2 image, and sought a consensus in cases of disagreement.

\section{Statistical analysis}

We compared the proportion of positive DWI scans between patients with a clinical diagnosis of TIA and of minor stroke. We also related the proportion of positive DWI scans to baseline clinical characteristics, the duration of symptoms and to the time since symptom onset and, in patients with minor stroke, to the persistence of symptoms and to the presence of clinical signs. Proportions were compared with the $\chi^{2}$ test. We related the presence of a lesion on DWI to the NIH stroke score both with the $t$ test and the Mann-Whitney test. We also used the non-parametric test because the patients had nondisabling strokes, and the NIH stroke scores would therefore not have been normally distributed. We related age to the presence of a lesion on DWI in a logistic regression analysis. These analyses were performed both unadjusted and adjusted for diagnosis (stroke versus TIA), sex, and NIH stroke score. Equally, diagnosis and NIH stroke score were also related to lesion presence on DWI in unadjusted and adjusted (age, sex, and diagnosis/NIH score as appropriate) logistic regression analyses. We related NIH score and age in a linear regression analysis. Interobserver and intraobserver reliability were determined with the $\kappa$ statistic. All statistical analyses were performed with SPSS version 10.0.

\section{RESULTS}

From September 2000 to April 2002257 patients in total attended the clinic. Of these, 68 were considered not to have 


\section{DWI}

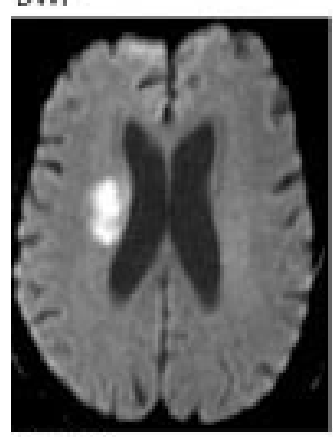

Potient 1

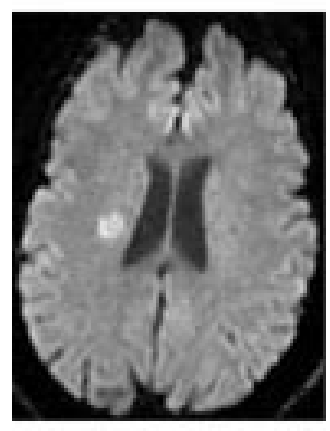

Potient 2
$A D C$
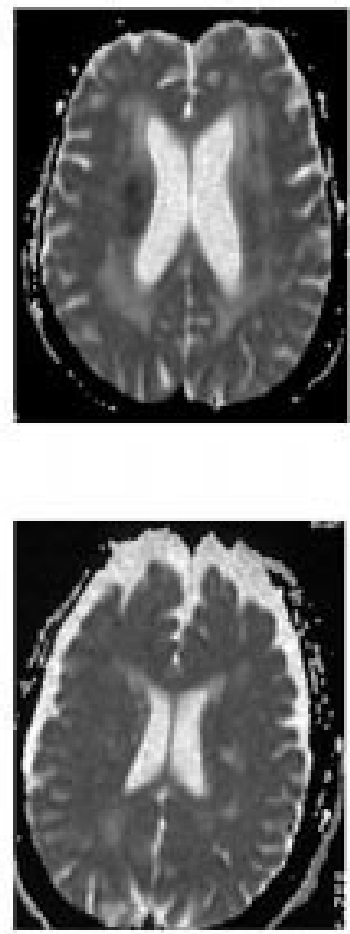

12

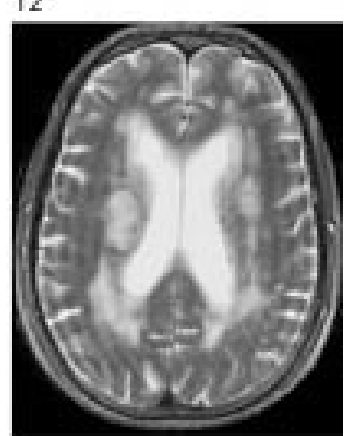

Figure 1 DWI scan, ADC map, and T2 weighted image for two minor stroke patients. Patient 1: 80 year old woman, dysarthria and left hemiparesis 14 days before, symptoms persisting. Note the low signal on the ADC map. Patient 2: 71 year old man with dysarthria, left sided weakness and sensory loss, onset 21 days before, symptoms persisting. The DWI signal is less hyperintense and the ADC has pseudo-normalised. had a cerebrovascular event. Of the 189 patients with a history suggestive of stroke or TIA, 11 patients did not undergo MRI (refused, claustrophobia, cardiac pacemaker), and five were found on MRI to have non-ischaemic disorders. Seventy two patients presented within two weeks of symptom onset and were excluded from the study. We therefore included 101 patients (53 men, mean age 70 years (SD 11)) with a clinical diagnosis of non-disabling stroke or TIA, who attended the clinic at least two weeks after their last symptomatic event (median time since event $=2 \mathrm{l}$ days, interquartile range (IQR) $=17-28$ ). Sixty seven patients attended between two and four weeks after symptom onset, and 34 patients attended with a delay of more than four weeks. Fifty one $(50.5 \%)$ patients had a clinical history of minor stroke and $50(49.5 \%)$ had a history of a TIA. Further baseline characteristics are shown in table 1.

Reproducibility of the identification of a clinically appropriate lesion on the DWI scan was high: both observers agreed in $97.1 \%$ of scans $(\kappa=0.93$; $95 \%$ confidence intervals $(95 \% \mathrm{CI})$ 0.83 to $1.00 ; \mathrm{p}<0.0001$ ), and for intraobserver agreement there was $93.9 \%$ agreement between the first and second scan assessment $(\kappa=0.88$; $95 \%$ CI 0.67 to $0.99 ; p<0.0001)$.

A clinically appropriate lesion (positive scan) was present on 36 of 101 DWI scans. In five of these, the ADC map still showed low signal intensity in the corresponding area. One of these patients had an ipsilateral severe carotid stenosis and several borderzone infarcts. The other four patients had single subcortical infarcts and severe leukoariosis. In 34 of the 36 cases with positive DWI scans, a corresponding lesion was visible on the T2 image. Examples of positive DWI scans and their corresponding $\mathrm{T} 2$ and $\mathrm{ADC}$ images are shown in figure 1. The proportion of positive scans was significantly higher in patients with minor stroke than in patients with TIA: $56.9 \%$ compared with $14.0 \%(p<0.0001)$. There were five patients with multiple lesions on the DWI scan. However, these were all located in the carotid artery territory of one hemisphere, there were no patients with lesions in multiple vascular territories.

The frequency of positive scans was unrelated to the time since the presenting event (fig 2). Of patients presenting two to four weeks after symptom onset, 20 of 35 minor stroke patients (57\%) and five of 32 TIA patients (16\%) had a positive DWI scan. Of patients presenting more than four weeks after symptom onset, 9 of 16 minor stroke patients (56.3\%) and 2 of 18 TIA patients (11.1\%) had a positive DWI scan (fig 2). For the TIA patients there was no statistically significant association between duration of symptoms and presence of a lesion on the DWI scan: 5 of 30 patients (16.7\%) with symptoms for less than an hour, 1 of 15 patients $(6.7 \%)$ with symptoms of between one and six hours duration, and 1 of 5 patients $(20.0 \%)$ with symptoms of between 6 and 24 hours duration showed a lesion on DWI $(p=0.21)$.

Of the 51 patients with minor stroke, $40(78.4 \%)$ still had symptoms attributable to the stroke at the time of presentation, and in $39(76.4 \%)$ patients a relevant neurological deficit

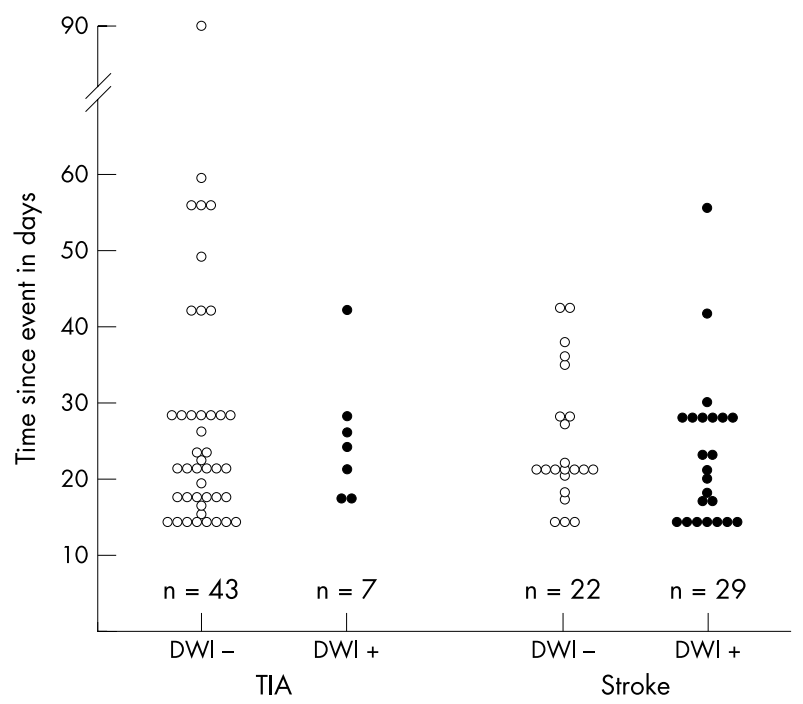

Figure 2 Days from symptom onset to clinic attendance/DWI scanning for 50 TIA patients and 51 stroke patients. For both groups the number of patients with positive and negative DWI scans (see text for definitions) is shown. 
was found on examination. At the time of clinic attendance the NIH score was 0 in 20 patients, $1-3$ in 26 patients, and 4 or more in five patients ( 4 in three patients, and 5 and 9 in one patient). The overall mean NIH stroke score was 0.85 $(\mathrm{SD}=1.47)$. Patients with a positive DWI had a significantly higher mean NIH score than patients with a negative scan: $1.75(\mathrm{SD}=1.93)$ compared with $0.35(\mathrm{SD}=0.78), \mathrm{p}<0.0001$, and the median NIH score was equally higher in patients with a positive DWI compared with patients with a negative DWI: 2 $(\mathrm{IQR}=0-3)$ compared with $0(\mathrm{IQR}=0-1), \mathrm{p}=0.01$. We found $\mathrm{a}$ borderline significant trend towards DWI being positive more frequently in patients with persisting signs ( 25 of 39; 64.1\%) compared with patients with a normal neurological examination ( 4 of $12 ; 33.3 \% ; \mathrm{p}=0.06$ ), and a similar trend was seen in patients with persisting symptoms ( 25 of $40 ; 62.5 \%$ ) compared with those who had made a full recovery from their stroke ( 4 of $11 ; 36.4 \% ; \mathrm{p}=0.12$ ).

Among the 101 patients with TIA or minor stroke, increasing age was associated with a positive DWI scan (mean age $=73.3$ years $(S D=8.3)$ for patients with positive scans compared with 67.8 years $(\mathrm{SD}=11.2), \mathrm{p}=0.01)$. This association remained significant after adjusting for sex and type of presenting event in a logistic regression analysis $(p=0.03)$, but was no longer significant after adjustment for NIH score $(p=0.07)$. NIH score and increasing age were positively correlated. Though weak, this correlation was statistically significant: $r=0.21, \mathrm{p}=0.038$. A history of previous TIA was a predictor of a negative DWI scan $(p=0.022)$. This association remained significant after adjusting for age and sex, but not after adjusting for diagnosis in a logistic regression analysis. There was a trend for patients presenting with a TIA to be more likely to have a history of previous TIA than patients presenting with a stroke: 13 of $50(26.0 \%)$ compared with 8 of $51(15.7 \%)$, respectively. However, this did not reach statistical significance $(p=0.202)$. Overall, the only statistically significant independent predictors of a positive DWI scan were presentation with minor stroke compared with TIA $(p=0.009)$ and increasing NIH score $(p=0.009)$. The relation between further clinical baseline characteristics and the presence of a lesion on DWI are shown in table 1.

Of the 36 patients with a positive DWI scan, four had had a previous stroke between one and 10 years previously. In all of these the lesion corresponding to the previous stroke was visible on the T2 scan, but in no patient was it visible on DWI. In addition, there were 25 patients with multiple lesions in different vascular territories on $\mathrm{T} 2$, suggesting previous cerebrovascular events. Only one of these had lesions in different territories visible on DWI, suggesting that DWI does not remain positive in the chronic phase.

\section{DISCUSSION}

In this study, DWI showed a clinically appropriate ischaemic lesion in $57 \%$ of minor stroke patients and $14 \%$ of TIA patients presenting more than two weeks after the symptomatic event. These data suggest that a high proportion of DWI lesions that are visible in the acute phase in patients with minor stroke persist beyond two weeks and may remain visible for up to two months. In contrast, although previous studies have reported high rates of appropriate DWI lesions in TIA patients scanned within a few hours or days of the event, ${ }^{78}$ our findings suggest that the majority of these lesions do not persist after two weeks.

High signal on DWI due to recent ischaemic lesions results from the combined effects of a reduced ADC and T2 shine through. ${ }^{9}$ ADC is usually reduced for one to two weeks after an ischaemic event and then pseudo-normalises and subsequently becomes increased, at which stage it leads to a reduced signal on DWI. ${ }^{9}{ }^{10}$ Simultaneously, T2 effects contribute to the high signal on DWI after the first few days, and overall high signal lesions on DWI in the late stages after an ischaemic event are due to T2 shine through effects rather than the ADC. As T2 weighted signal remains increased in chronic ischaemic lesions, this implies that the signal of DWI lesions with a high proportion of T2 shine through should remain high for a long time. However, only few data exist on the time course of DWI lesions beyond the acute stage. A small study has suggested that DWI signal may remain high for several weeks, and that it can still change more than three months after an event. ${ }^{9}$ In our study, we were unable to study the time course of the DWI lesions. However, the fact that several weeks after a minor stroke $57 \%$ of patients had a lesion on $\mathrm{T} 2$ and a corresponding lesion on DWI, whereas virtually none of the older T2 lesions were visible on DWI, suggests that the DWI signal still changes more than a few weeks after an ischaemic event. As our study was cross sectional and we also do not know the age of the T2 lesions, this finding requires confirmation in longitudinal studies. However, it suggests that DWI may be helpful in differentiating subacute from chronic cerebral ischaemic lesions.

A disadvantage of the cross sectional design is that because at least two weeks had elapsed between symptom onset and scanning, we cannot be certain that all of the lesion(s) seen on DWI definitely represented the last symptomatic event-to achieve this, a follow up study from symptom onset would have been required. However, we only classified scans as positive if the site of the lesion(s) matched the clinical presentation, and we calculated the age of a lesion according to the last symptomatic event rather than the original presenting event, although these were usually identical. Finally, the ADC appeared normal or high in the large majority of patients with a high signal lesion on the blo00 image, indicating that this was probably mostly attributable to T2 shine through effects and therefore older than 10-14 days. ${ }^{10}$ There were five patients in whom the ADC had remained low for more than 14 days after the event. This may have been attributable to an intercurrent asymptomatic infarct. However, a persistently low ADC more than two weeks after an ischaemic event has also been reported by others. ${ }^{9}{ }^{14} 15$ One study found that in patients with haemodynamic watershed infarcts the ADC remained low for much longer than in patients with territorial, presumed thromboembolic infarcts-it often normalised only one month after symptom onset. ${ }^{15}$ In our study, only one of the five patients with a persistently low ADC had a watershed infarct. The other four had severe leukoariosis. It has been shown that the ADC in leukoaraiotic white matter is higher compared with normal white matter. ${ }^{17}$ It may simply take longer for the ADC of an infarcted region to reach the higher ADC of the surrounding or contralateral leukoaraiotic tissue, and the infarct therefore remains visible for longer. Alternatively, the leukoaraiosis may reflect chronic hypoperfusion and the persistently low ADC may be caused by similar mechanisms as in the patients with watershed infarction.

A disadvantage of the study was that in the presence of negative imaging data, it can be difficult to be certain of a diagnosis of ischaemic stroke or TIA. However, all the patients included in this study were felt by two neurologists to have had a diagnosis of ischaemic stroke or TIA, and patients with other diagnoses were excluded. It is still possible that some patients who had not had a TIA or minor stroke were included in the study. DWI in these patients would be negative, and including them would therefore underestimate the sensitivity of DWI. A further difficulty is the differentiation between stroke and TIA. The time cut off of 24 hours for TIAs is arbitrary, and most TIAs last much less than 24 hours. In our study, $62 \%$ of the TIAs lasted less than an hour, and only $10 \%$ longer than six hours. Despite this short duration, DWI lesions were present in $14 \%$ of TIA patients. The rate of positive scans did not differ according to symptom duration, but numbers were small. In these cases the differentiation between TIA and stroke may be of little benefit, and establishing the diagnosis 
of a cerebral ischaemic event irrespective of its duration is more helpful. However, in the absence of a positive scan, the clinical differentiation between stroke and TIA may still be helpful, although the 24 hour cut off remains arbitrary.

In this study, we found that increasing age was associated with a positive DWI scan, although this association was no longer present after adjusting for stroke severity (NIH score). It has been reported that the time course of the ADC may differ in the first few days after a stroke depending on patient age. ${ }^{17}$ The authors speculated that this could be attributable to different rates of spontaneous recanalisation and presence of collateral vessels in young compared with older patients. ${ }^{17}$ It may be that this is partially reflected in our findings as well. However, we also found a trend for increasing age to be associated with a higher NIH score. After adjusting for NIH score, age and DWI positivity were no longer associated, suggesting that the higher rate of positive scans in older patients may simply be attributable to their having more severe strokes, which may cause more obvious lesions on DWI.

Our analyses showed that a history of previous TIA was a predictor of a negative DWI scan. This association disappeared after adjusting for diagnosis of stroke versus TIA, and there was a trend for a history of previous TIA to be associated with the presenting event being a TIA. The association between previous TIAs and negative DWI scans therefore probably simply reflects the fact that patients with a history of TIA are more likely to have further TIAs ${ }^{18}$ and, as we have shown, the rate for positive scans in TIA patients is low.

In summary, this study shows that DWI is still reasonably sensitive in patients with minor stroke scanned more than two weeks after the last symptomatic event, and that radiological assessment at that stage has good interobserver and intraobserver reproducibility. It may therefore be useful in routine clinical practice in patients presenting late, and further studies are justified. In contrast, the high rates of appropriate DWI lesions reported in TIA patients scanned within a few hours or days of the event are not seen in patients presenting more than two weeks after the event.

\section{Authors' affiliations}

U G R Schulz, P M Rothwell, Stroke Prevention Research Unit, Department of Clinical Neurology, Radcliffe Infirmary, Oxford, UK D Briley, Department of Neurology, Stoke Mandeville Hospital NHS Trust, Aylesbury, UK and Department of Neurology, Radcliffe Infirmary
T Meagher, Department of Radiology, Stoke Mandeville Hospital NHS Trust

A Molyneux, Department of Neuroradiology, Radcliffe Infirmary

\section{REFERENCES}

1 AHA Medical/Scientific Statement. Guidelines for the management of transient ischemic attacks. Stroke 1994;25:1320-335.

2 Dennis M, Bamford J, Sandercock P, et al. Prognosis of transient ischaemic attacks in the Oxfordshire Community Stroke Project. Stroke 1990;21:848-53.

3 Jorgensen HS, Nakayama H, Reith J, et al. Factors delaying hospital admission in acute stroke: the Copenhagen Stroke Study. Neurology 1996;47:383-7.

4 Goldstein LB, Edwards MG, Wood DP. Delay between stroke onset and emergency department evaluation. Neuroepidemiology 2001;20:196-200.

5 Moseley ME, Cohen Y, Mintorovitch J, et al. Early detection of regional cerebral ischemia in cats: comparison of diffusion- and T2-weighted MRI and spectroscopy. Magn Reson Med 1990;14:330-46.

6 Albers GW. Advances in intravenous thrombolytic therapy for treatment of acute stroke. Neurology 2001;57:S77-81.

7 Kidwell CS, Alger JR, Di Salle F, et al. Diffusion MRI in patients with transient ischemic attacks. Stroke 1999:30:1174-80.

8 Rovira A, Rovira-Gols A, Pedraza S, et al. Diffusion-weighted MR imaging in the acute phase of transient ischaemic attacks. ANNR 2002;23:77-83.

9 Geijer B, Lindgren A, Brockstedt S. Persistent high signal changes in diffusion weighted MRI in late stages of small cortical and lacunar ischemic stroke lesions. Neuroradiology 2001;43:115-22.

10 Lansberg MG, Thijs VN, O'Brien MW. Evolution of apparent diffusion coefficient, diffusion weighted and T2 weighted signal intensity of acute stroke. Am J Neuroradiol 2001;22:637-44.

11 Augustin M, Bammer R, Simbrunner J. Diffusion weighted imaging of patients with subacute cerebral ischemia: comparison with conventional and contrast enhanced MR imaging. Am J Neuroradiol 2000;21:1596-602.

12 World Health Organisation. Cerebrovascular diseases - prevention, treatment and rehabilitation. Technical Report Series no 469. Geneva: WHO, 1971 .

13 Brott T, Adams HP Jr, Olinger CP, et al. Measurements of acute cerebral infarction: a clinical examination scale. Stroke 1989;20:864-70.

14 Schwamm LH, Koroshetz WJ, Sorensen AG, et al. Time course of lesion development in patients with acute stroke: serial diffusion- and haemodynamic-weighted magnetic resonance imaging. Stroke 1998;29:2268-76.

15 Huang IJ, Chen CY, Chung HW, et al. Time course of cerebral infarction in the middle cerebral artery territory: deep watershed versus territorial subtypes on diffusion weighted MR images. Radiology 2001;221:35-42.

16 Helenius J, Soinne L, Salonen O, et al. Leukoaraiosis, ischemic stroke, and normal white matter on diffusion-weighted MRI. Stroke 2002;33:45-50.

17 Copen WA, Schwamm LH, Gonzalez RG, et al. Ischemic stroke: effects of etiology and patient age on the time course of the core apparent diffusion coefficient. Radiology 2001;221:27-34.

18 Weimar C, Kraywinkel K, Rodl J, et al. Etiology, duration, and prognosis of transient ischemic attacks: an analysis from the German Stroke Data Bank. Arch Neurol 2002;59:1584-8. 